\title{
Towards Self-Adaptive Monitoring Framework For Integrated Management
}

\author{
Audrey Moui, Thierry Desprats \\ IRIT, Université Paul Sabatier \\ 118 route de Narbonne, 31062 Toulouse cedex 9, France
}

\begin{abstract}
Integrated management copes with management of heterogeneous and complex systems in a multi-level dimension (network, systems, services). In this context, monitoring activity has to be concerned with efficiency and autonomy. This paper presents work in progress to define a framework for self-adaptive monitoring relying on the characterization of governability, adaptability and configurability.
\end{abstract}

\section{Motivation}

The multi-level dimension of integrated management (network, systems, services), the dependence between heterogeneous components and the affluence of management information make the management activity more and more difficult to perform. Currently, management is performed with the help of the classical MAPE loop [2]: the managed system is observed (M) and an analysis (A) is made to detect failures or every particular or relevant situations. If there are any, a technical decision is planned $(\mathrm{P})$ and executed $(\mathrm{E})$ on the system to improve its efficiency. The monitoring activity is a central point in integrated management. Indeed, monitoring aims at collecting from the various system components a set of relevant data: thus, its state and its behaviour can be known. Moreover, the monitoring activity makes it possible to detect all the perturbations occurring on the whole system or on one of its part. The monitoring can also have different purposes, like management optimization, protection, control, accounting or even maintenance.

But what are the requirements to be positioned on the collected data? Is it necessary to monitor everything, all the time, from all the components of the system? What is the importance of the volume of data to be stored and processed? How is it possible to select which data are useful, relevant, to be monitored? Have some quality criteria like accuracy, freshness, correctness to be taken into account or is it possible to collect data with a certain degree of uncertainty? May suitable or efficient decisions be taken with an important part of fuzzy or imprecise knowledge on the managed composite system state? Is the monitoring activity able to consider integrations and scalability of the managed domain? Mechanisms like event correlation, filtering and log can help dealing with the collected data: are they used by the current monitoring activity in the most efficient way? 
Finally, other contraints have also to be taken into account: system needs to be monitored at runtime, and the availability and the capacity of the technological ressources which support the execution of monitoring can constitute a restriction (storage, energy level, CPU load, bandwidth, etc ).

So, we need to think about how improving the adaptiveness level of monitoring activities. Monitoring is required to become more flexible to autonomously tackle, in a relevant and efficient way, the variation of both "business" requirements and environmental constraints.

Numerous works already contributed to make the monitoring activity adaptable (e.g. [1] is not generic enough to be used in every management context this one is based on SNMP -; RAP [4] is only concerned with polling adaptability; Ganglia [3] provides the ability to reconfigure the monitoring mechanisms according to the network context - but only for small networks). Note also that all these approaches only focused on network management, not on integrated management.

\section{An Analysis To Deal With The Adaptability}

Self-adaptive monitoring implies a self-governed and automatic behaviour adaptation of monitoring activity. The monitoring activity is a process which is based on the use, possibly combined, of polling and event reporting mechanisms.

The underlying mechanisms, and the way they are operated, must consider the high level business objectives. They have to provide relevant inputs for the analysis activity (why do we monitor?). Determining the nature of these mechanisms (how?), the managed elements they concern (what?) and the temporal mode by which they operate (when?) constitutes what we defined as a "monitoring strategy".

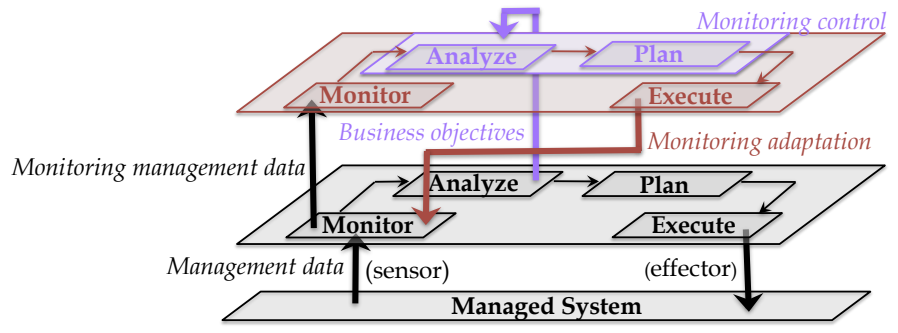

Fig. 1. The Adaptive Monitoring Framework

Our approach aims at creating a framework which would be able to support automatically the adaptation of the monitoring activity. An adaptation will result in a monitoring strategy change, and consequently will modify the monitoring activity behaviour. Automating monitoring adaptation requires to manage and control the monitoring activity. It becomes necessary to introduce 
a second management loop to control the monitoring. As shown in Figure 1, for this loop, the managed system is the monitoring activity itself. We identified the three following levels of capabilities to be managed in order to perform on line monitoring adaptation:

1. The ability to detect a need of monitoring adaptation (Governability): this is the role of the "Monitoring control" plan (in purple) to decide if and how the monitoring activity has to be adjusted.

2. The ability to execute (in red) the monitoring adaptation (Adaptability) which has been decided by the "Monitoring control" plan, by performing operations on the set of mechanisms, with the objective to modify the current monitoring strategy.

3. The capacity of the monitoring mechanisms to be dynamically adapted (Configurability): parameters governing the behaviour of each monitoring mechanism can be modified dynamically at runtime and without disruption.

\section{Global Approach And Work-in-progress}

Figure 2 depicts the global approach which governs the progression of our works:

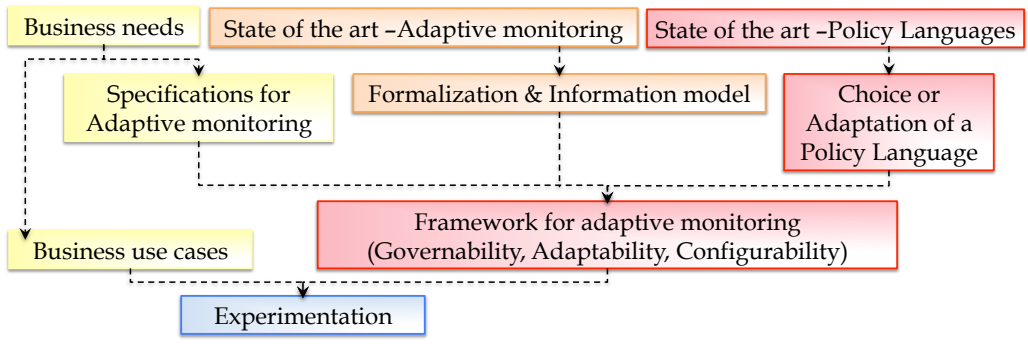

Fig. 2. Approach To Make Adaptable The Monitoring

The left axe (in yellow) is concerned with what we called "business requirements" and aims at bringing, in addition to several use case identification, the logics for determination and adaptation of a business-oriented monitoring strategy. The outputs stay at a high level of knowledge and has to be translated into more technical rules. They represent the content used by our "Monitoring control" plan.

The formalization of this logic (middle orange axe) is part of the current work. It is concerned with both the definition of generic management information models and the operators which will support the enforcement of a monitoring adaptation decision. They have to be generic enough to be used for business context adaptation requirements, or for monitoring self-optimization, or for adaptation in response to some constraints variations on technological 
ressources availability. The management information model scope is concerned at the same time with the configurability of the basic monitoring mechanisms (polling and event reporting) and with the monitoring strategy manageability. The enforcement operators allow the creation, deletion or reconfiguration of one or more mechanisms belonging to a monitoring strategy.

Moreover knowledge is necessary to take a monitoring strategy adaptation decision. Therefore, the framework must provide language facility to express, for one adaptation, both the triggering conditions and the resulting actions to be enforced. Thus, future works (right axe) will be concerned with the study, as a candidate approach, of event/conditions/actions-based management policies.

Finally, we will experiment concepts and derived tools of this framework on some business use cases.

\section{Conclusion and Perspectives}

Self-adaptive monitoring is an approach that aims at making the monitoring the less intrusive possible, by efficiently adjusting itself at runtime to every situations variation. This paper describes a work in progress which aims to obtain a generic model-based framework able to support the manageability of monitoring strategies. This approach relies on the characterisation of three complementary levels which respectively deal with configurability of basic monitoring mechanisms, adaptability and governability of monitoring strategy. Management information models and changing operators are defined to support adaptability automation. This automation will reduce the human operations over the system with an increase of effectiveness and fastness, and with an optimized adjustment of the monitoring activity. Some parts of this work have already been implemented on a CIM-WBEM environment [5]. Further works will focus on the governability level before experimenting the framework on a specific business environment where adaptive monitoring is required to improve real time diagnostic support in a case of integrated management.

\section{References}

1. Duarte Jr, Elias Procópio; Musicante, Martin A.; Fernandes, Henrique H.: ANEMONA: a Programming Language for Network Monitoring Applications. Internat. Journ. of Net. Managem.. 18(4) (August 2008).

2. Kephart, Jeffrey O.; Chess, David M.: The Vision of Autonomic Computing. Computer. 36(1) (January 2003) 41-50.

3. Massie, M.L.; Chun, B.N.; Culler, D.E.: The Ganglia Distributed Monitoring System: Design, Implementation, and Experience. Parallel Computing. 30(7) (2004) 817-840.

4. Moghé, P.; Evangelista, M.H.: RAP - Rate Adaptive Polling for Network Management Applications. in Proceedings of IEEE Network Operation and Management Symposium NOMS'98. (1998) 395-399.

5. Moui, Audrey; Desprats, Thierry; Lavinal, Emmanuel; Sibilla, Michelle: Managing Polling Adaptability in a CIM/WBEM Infrastructure. Int. DMTF Workshop on Systems \& Virtualization Managt. (SVM10) (2010) 1-6. 